ROCZNIKI TEOLOGICZNE

Tom LXVII, zeszyt 3 - 2020

DOI: https://doi.org/10.18290/rt.20673-2

\title{
DIMENSION SPIRITUELLE EN ÉVIDENCE DANS LE DOMAINE DE LA SANTÉ PAR LA PROFESSION DES INTERVENANTS EN SOINS SPIRITUELS (EXEMPLE DU QUÉBEC)
}

\author{
SPIRITUAL DIMENSION HIGHLIGHTED IN THE FIELD OF HEALTH \\ BY THE PROFESSION OF SPIRITUAL CARE WORKERS (QUEBEC EXAMPLE)
}

\begin{abstract}
Many societies throughout the world, formerly deeply Christian, have become secular in recent decades. Quebec society is no exception, and the reasons for this are diverse. We can see how schools and hospitals once run by religious orders have become state property. In the past, the Catholic Church played an important role in society and took care of spiritual aspects of its people. Presently, it no longer provides these services. But this does not mean that people's spiritual needs and their thirst for transcendence have disappeared. Needs for an overall balance of life often manifest themselves in a crisis caused by illness. It is especially true in crucial moments, such as suffering, illness or closeness to death, that people ask many questions about spirituality.

A positive aspect is that Quebec's secular society respects the rights of individuals and their spiritual needs. To meet these needs, a new profession has emerged and it highlights the spiritual dimension of people. It must be taken into account by all those committed to giving support to the sick. The person as a whole "soul-body" must be at the centre during all medical care. It is for this reason that in this article I spoke firstly about the concept of the person in which the holistic and integrative approach prevails.

I then focused on the notion of spirituality. The spiritual sphere is difficult to define. By analyzing the different notions of spirituality, there are several common elements, such as seeking answers to the following questions: the identity of a human being ("who am I?"), the purpose and meaning of life ("why do I live"), the meaning of suffering and death ("Why does this happen to me?"). The problem of the meaning and purpose of life is a primordial question for man, even if it is often unexpressed. Many people find the answers to these questions in
\end{abstract}

Dr HALYNa Kryshtal - Institut de Formation Théologique de Montréal (Kanada); address: 2065, rue Sherbrooke Quest Montréal (QC), H3H 1G6, Canada; e-mail: h.kryshtal@ iftm.ca 
religion, in their relationship with transcendence / Supreme Being / God. Some people, however, consider spirituality in complete isolation from religion. Then, the main elements of the spiritual sphere are often relations with relatives and human values. Spiritual care workers help those suffering to regain the meaning of life, rebuild a global balance of life and/or learn to "live with" the limits. Through their profession they offer attentive listening, the possibility of dialogue, solidarity, compassion and love that the person so needs.

It is noteworthy that theological faculties and institutes of religious studies have reoriented themselves to offer programs to train spiritual care workers.

Key words: integral vision of the human person; spirituality; health; spiritual support; spiritual care workers; suffering; illness, meaning of life; human values.

\section{INTRODUCTION}

La fameuse phrase selon laquelle «le XXIe siècle sera spirituel ou ne sera pas» est très présente dans l'esprit des gens et trouve un écho dans différents milieux. On l'attribue à l'écrivain français André Malraux ${ }^{1}$. Nous, qui vivons au début du XXI siècle, pouvons présentement facilement constater un grand intérêt pour la notion de «spiritualité». Ce concept est traité par différents type de sciences: la philosophie, la théologie, la psychologie, la sociologie, l'ergothérapie, la médecine, etc.

Justement dans les dernières décennies nous pouvons observer un vif intérêt pour les questions de spiritualité dans le domaine de la santé. Diverses publications nous témoignent de cela. Nous nous demandons quelles sont les causes de cette «re-connaissance» ou encore de cette «re-découverte» de la dimension spirituelle de l'humain, et quel lien existe entre cette dimension spirituelle et la santé ${ }^{2}$ ? D'où vient cet intérêt et pourquoi il se prononce-il avec une telle intensité? Une des réponses à cette question est donnée dans l'article de N. Simard: «[...] la grande majorité des recherches scientifiques sur l'influence de la spiritualité dans la santé appuient l'idée que les valeurs spirituelles et les buts de la vie apportent une contribution indéniable à la santé physique et psychique ainsi qu'à la satisfaction de vivre. Pour une conception globale de la santé - ce que la médecine scientifique moderne a négligé -, il faut une intégration de la dimension spirituelle dans les soins

1 L'histoire en citation, https://www.histoire-en-citations.fr/citations/le-xxie-siecle-seraspirituel-ou-ne-sera-pas (16.04.2020).

${ }^{2}$ J. PEPIN, Ch. CARA, La réappropriation de la dimension spirituelle en sciences infirmières, „Théologiques”, vol. 9, $\mathrm{n}^{\circ} 2,2001$, p. 33. 
de la santé» ${ }^{3}$. L'autre explication peut être «la reconnaissance des limites de la médecine scientifique [...] ne peut résoudre le scandale de la souffrance et $[\ldots]$ semble avoir réduit le patient à un objet d'analyse et de recherche» ${ }^{4}$.

Tous ceux qui sont liés au domaine de santé doivent essayer de comprendre ce que la spiritualité, comme telle, a en soi et aussi considérer cette dimension spirituelle de la personne dans le processus de la prévention ou de la guérison de la maladie. De plus, les intervenants en soins spirituels qui sont appelés à porter le soulagement spirituel aux personnes malades, doivent avoir des idées claires sur la spiritualité et de son importance dans le domaine de la santé. C'est d'ailleurs par la profession des intervenants en soins spirituels que cette dimension spirituelle retrouve son exposition même au monde très laïcisé.

De là le titre du présent article: «Dimension spirituelle en évidence dans le domaine de la santé par la profession des intervenants en soins spirituels». Je vais d'abord présenter le contexte de l'apparition de cette nouvelle profession au Québec. Par après, je vais parler du concept de la personne et de la notion de la spiritualité qui est étroitement liée à la personne. Ensuite, je vais aborder le concept de la santé et surtout ses liens avec la spiritualité. Je terminerai par des réflexions sur les soins spirituels qui peuvent être donnés par les intervenants ou les intervenantes dans le domaine de la santé. Dans cet article, je vais me baser sur les différentes sources publiées qui clarifient ma position. Il est important d'ajouter que toutes ces notions sont bien complexes et qu'on ne pourrait en expliquer que des traits principaux.

\section{CONTEXTE D'APPARITION DE LA PROFESSION DES INTERVENANTS EN SOINS SPIRITUELS AU QUÉBEC}

L'intervention en soins spirituels est une profession qui a apparu au Canada assez récemment. Pour mieux comprendre son évolution, il faudrait considérer les changements qui ont eu lieu dans les dernières décennies au Québec. Surtout il ne faut pas oublier la Révolution tranquille des années '60. Une de ces conséquences est l'affaiblissement du rôle de l'Église catholique dans la société québécoise. Autrefois, c'était les aumôniers ou les animateurs de la pastorale, mandatés par les ordres religieux et les diocèses, qui ren-

\footnotetext{
${ }^{3}$ N. SimARD, Spiritualité et santé, „Reflets”, vol. 12, n 1, 2006, p. 107.

${ }^{4}$ N. SIMARD, Spiritualité et santé, p. 114.
} 
daient service aux hôpitaux en offrant un réconfort spirituel aux personnes malades et à leurs familles. Depuis que la société est devenue de plus en plus laïque, les aumôniers ont été remplacés par les intervenants en soins spirituels. Il faut préciser que cette profession s'est développée dans le cadre de la loi du Québec qui parle de «services de santé ou sociaux respectueux des droits des personnes et de leurs besoins spirituels» (art. 100) ${ }^{5}$.

Cette profession n'est plus liée à une religion particulière. Elle peut être exercée par des représentants de diverses cultures et religions. Cela répond à la réalité de la société canadienne actuelle qui est multiculturelle et multiou encore non-religieuse. Des livres sur les religions et la santé, dans lesquels on peut trouver des références pratiques pour les intervenants et intervenantes en milieux de santé ne manquent pas. Il s'agit, bien évidemment, de donner le soutien à toute personne, qu'elle soit croyante ou non, dans les différents secteurs de soin $^{6}$.

Pour effectuer cette profession, il faut aimer être en contact avec les personnes, avoir une facilité à communiquer et une capacité d'écoute, être capable de s'adapter à tous les types de milieux et de clientèles, et bien sûr, savoir observer et analyser les situations et les personnes ${ }^{7}$. Ce type de travail exige une personnalité mature, une sensibilité aux besoins des autres, mais aussi d'avoir une formation universitaire appropriée. Au Québec, il existe divers programmes de formation en théologie, en pastorale ou en sciences religieuses offerts dans les universités et les collèges religieux. En répondant aux signes des temps, les facultés ou les départements de théologie se sont réorientés pour offrir la formation des prochains intervenants en soins spirituels.

\section{CONCEPT DE LA PERSONNE}

Pour la profession des intervenants en soins spirituels le concept de la personne est fondamental. Tous les autres concepts se croisent à sa base. Ce con-

5 Loi sur les services de santé et les services sociaux, http://legisquebec.gouv.qc.ca/fr/sho $\mathrm{wdoc} / \mathrm{cs} / \mathrm{s}-4.2$.

${ }^{6}$ Pour mieux comprendre le contexte québécois, je conseille l'article suivant: Jacques Cherblanc et Guy Jobin, Soutenir le développement du pouvoir d'agir: une composante essentielle des soins spirituels au Québec, „Studies in Religion/Sciences religieuses”, pp. 1-24, https://constellation.uqac.ca/5462/1/Article\%20Empowerment\%20ISS\%5Bfinal\%5D_sans\%20 marque.pdf

7 Loi sur les services de santé et les services sociaux, http://legisquebec.gouv.qc.ca/fr/sho wdoc/cs/s-4.2. 
cept est en même temps bien complexe. Depuis ses origines l'humanité fait un effort à bien connaître et définir ce qu'est l'être humain. À travers les siècles les scientifiques discutent de ce sujet en mettant en évidence l'un ou l'autre aspect de la personne. L'aspect physique de la personne est plus facile à explorer. La biologie peut tout raconter de l'histoire naturelle des êtres vivants, y inclus les êtres humains. La médecine explore le corps humain, son fonctionnement normal et cherche à préserver la santé par la prévention et le traitement des pathologies. La science moderne, telle que la biologie moléculaire, examine le code génétique de la cellule, du tissu et de la programmation de la vie physique. Pourtant, il y a toujours quelque chose qui nous échappe et qui nous pousse à découvrir un élément non-matériel dans la personne. Ainsi, il suffit de mentionner Carl Jung, Alfred Adler, William James et Sigmund Freud pour comprendre que le dernier siècle nous a offert des études en psychologie bien approfondies. Étymologiquement, le mot psychologie dérive du latin psychologia, terme lui-même formé à partir du grec ancien $\psi v \chi \eta ் ~(p s u k h \bar{e})$ qui signifie «le souffle, l'esprit, l'âme» ${ }^{8}$.

Il est donc évident qu'on ne peut pas réduire la personne aux facteurs matériels. Il y a quelque chose en elle qui dépasse et transcende le corps physique. Déjà Philon d'Alexandrie, au Ier siècle après Jésus-Christ, disait que pour le thérapeute, le corps de la personne malade ne peut jamais être vu comme un objet, comme une chose ou une machine défectueuse que le médecin doit «réparer». Le corps est un corps «animé» ${ }^{9}$. Une des expressions latines disait «corpore et anima unus», c'est-à-dire que le corps et l'âme de la personne sont unis et constituent un être humain ${ }^{10}$. Il est donc important de voir la personne dans son intégralité, si bien exprimé dans la citation de la dixième Satire de Juvénal: «Mens sana in corpore sano» (un esprit sain dans un corps sain), qui souligne l'importance de prendre soin de sa santé mentale, de son âme, ainsi que de son corps ${ }^{11}$.

Certaines études vont encore plus loin et montrent la distinction entre l'âme et l'esprit, autrement dit un ternaire humain: «corps-âme-esprit». Selon M. Fromaget le mot «âme» signifie «le mental, la part psychique de l'être». «L'esprit» assimile des qualités ou fonctions psychiques telles la pensée,

${ }^{8}$ Définition: psychologie, http://www.psychomedia.qc.ca/lexique/definition/psychologie (22.04.2020).

${ }^{9}$ T. CHÂTEL, Prise en compte du spirituel et nouvelles représentations du soin, „Médicine palliative", n³, 2007, p. 196.

10 «Corpore et anima unus»: la rilevanza etica dell'unità sostanziale dell'uomo all'inizio del terzo millennio, Roma, 24 settembre 2003, http://www.caffarra.it/roma0903.php

${ }^{11}$ Juvénal, Satires, https://sites.google.com/site/texteschoisis/home/juvenal (22.04.2020). 
l'intelligence, ou bien l'ensemble des facultés psychologiques de la personne. De plus, «l'esprit» pour cet auteur est une réalité mystérieuse, spécifiquement religieuse ou spirituelle ${ }^{12}$.

L'anthropologie ternaire («corps-âme-esprit») est plus complète, plus «holistique», et peut fournir une base pertinente pour l'intervenant en soins spirituels qui travaille dans le domaine de santé. Dans le soin spirituel, il faut appliquer cette approche intégrative et globale, car il est approprié à la personne. Il est nécessaire de considérer le patient dans son ensemble en envisageant plusieurs dimensions de son être: physique, émotionnelle, intellectuelle et spirituelle ${ }^{13}$. Cette approche doit caractériser toutes les actions pour préserver la santé de la personne ou pour traiter ses maladies. Quant à la prévention de la maladie, je partage l'idée exprimée par C. Berghmans: «vu que l'individu est l'acteur de sa santé, il faut sensibiliser la personne à son propre rôle dans son équilibre et dans le maintien de sa santé» ${ }^{14}$. D'abord, l'être humain est invité à prendre soin de lui-même pour prévenir lapparition de maladies en faisant du sport, en étant attentif à l'alimentation et en gardant l'équilibre mental et psychique. Bien sûr, personne ne peut être assuré qu'en faisant tout ça il ne tombera pas gravement malade. Heureusement, grâce aux progrès technologiques et scientifiques, la médecine est capable de trouver des solutions à la plupart des maladies et des maux qui accablent l'humanité, et ce dans certains cas depuis ses débuts ${ }^{15}$.

L'intervenant en soins spirituels doit toujours se rappeler que le rôle de l'individu dans le processus de sa guérison ne diminue jamais. Pour soutenir cette position, il suffit de rappeler certaines observations faites dans le domaine de la santé. Le personnel médical se trouve toujours devant un mystère: deux personnes avec la même maladie et le même traitement auront-elles les mêmes résultats? L'une d'elles pourrait guérir complètement et l'autre pourrait mourir. Il doit y avoir alors quelque chose de plus qui influence les résultats. Ce ne sont pas seulement les médicaments qui peuvent aider à guérir le patient. Ce sont aussi ses activités cognitives, ses pensées, ses états

${ }^{12}$ M. Fromaget, De la distinction âmelesprit, autrement dit du ternaire humain. 2012, https://www.unidivers.fr/de-la-distinction-ameesprit-autrement-dit-du-ternaire-humain/ (version PDF, p. 2); J. PEPIN, Ch. CARA, La réappropriation de la dimension spirituelle en sciences infirmières, p. 33.

${ }^{13}$ C. Berghmans, J.-L. TORRes, Spiritualité et santé: un pont thérapeutique, „Intereditions" 2012, p. 15-16.

${ }^{14}$ C. Berghmans, J.-L. Torres, Spiritualité et santé: un pont thérapeutique, p. 10.

${ }^{15}$ C. Berghmans, J.-L. Torres, Spiritualité et santé : un pont thérapeutique, p. 10. 
d'esprit, sa motivation, ses croyances, son désir de guérir qui influencent directement son corps ${ }^{16}$. Dans ces cas-là, les liens entre le corps et l'esprit sont mis bien en évidence. Ainsi nous entrons dans la dimension de la spiritualité qui est reconnue comme un facteur contribuant à la santé chez beaucoup de gens.

\section{CONCEPT DE LA SPIRITUALITÉ}

Le mot esprit vient du latin «spiritus» qui signifie souffle ou vent. Ce mot dérive du verbe latin «spirare» - «souffler». Pour les personnes religieuses, l'esprit est un souffle de Dieu, l'inspiration provenant de Dieu. Pour les personnes non religieuses, l'esprit est la totalité des facultés mentales: perception, affectivité, intuition, pensée, concept, jugement, morale. La spiritualité est une notion qui a accompagné l'être humain depuis ses origines ${ }^{17}$. On perçoit l'esprit comme le principe de vie, «la dimension centrale de l'être humain» ${ }^{18}$. Donc, c'est quelque chose d'essentiel dans la vie de la personne. On peut associer la spiritualité à presque tout ce que désire le cœur humain $^{19}$. C'est peut-être pour cette raison qu'il est tellement difficile de donner une définition uniforme de la spiritualité.

Certains relient la spiritualité à la religion. En effet, ces deux notions peuvent être liées, mais elles ne sont pas identiques. Beghmans a fait l'allusion que «la spiritualité est une sœur jumelle de la religion» ${ }^{20}$. D'après T. Châtel le spirituel et la religion sont deux notions qui « se distinguent au fond, quoiqu'elles puissent aussi heureusement se combiner. On peut dire que, par principe, il y a du spirituel dans la religion mais que la religion n'est pas le seul vecteur du spirituel. Philosophie, psychologie, nouvelles spiritualités, morale, sacré, symbolisation, magie, humanisme [...]. et même athéisme sont

${ }^{16}$ C. Berghmans, J.-L. Torres, Spiritualité et santé: un pont thérapeutique, p. 8; C.M. PuChalski, Religion, Medicine and Spirituality: What We Know, What We Don't Know and What We Do, ,Asian Pacific Journal of Cancer Prevention”, vol. 11, 2010: MECC Supplement, p. 47.

17 C. Berghmans, J.-L. TORres, Spiritualité et santé: un pont thérapeutique, p. 10.

18 P.A. Potter, A.G. Perry, Soins infermières. Fondements généraux, vol. 1, Montréal 2009, p. 406.

${ }^{19} \mathrm{U}$. KING, La quête spirituelle à l'heure de la mondialisation, Québec 2010, p. 12.

${ }^{20}$ C. Berghmans, J.-L. TORRes, Spiritualité et santé: un pont thérapeutique, p. 37. 
autant de voies qui peuvent se prétendre, a différents égards, également porteuses de spirituel» ${ }^{21}$.

En suivant les réflexions de T. Châtel, dans le domaine de la santé, il faut toujours prendre en considération que tout être humain a une dimension spirituelle. La personne peut aussi choisir, ou non, d'avoir une religion et vivre sa religion de manière spirituelle. Mais, il est possible d'être spirituel sans religion. Ces deux possibilités sont visibles dans la définition de A. ConteSponville. Il définit la spiritualité comme «la partie de notre vie intérieure qui a un rapport avec l'absolu, l'infini ou l'éternité, que ceux-ci soient conçus comme transcendants et personnels (on parle alors de divinité et de Dieu) ou bien comme immanents et impersonnels (ou l'on parlera plutôt de l'être, de la vérité ou de la nature), avec bien sûr tous les degrés intermédiaires entre ces deux pôles» ${ }^{22}$.

Effectivement, la spiritualité est un concept à deux dimensions: la dimension horizontale ou existentielle qui touche les valeurs inhérentes aux relations humaines et aux activités qui donnent le sens à la vie, et la dimension verticale ou religieuse qui fait référence à une relation avec la transcendance ou avec un Être suprême. Certaines personnes s'intéressent presqu'exclusivement à la relation avec l'infini et oublient de cultiver la dimension existentielle ou horizontale de la spiritualité. D'autres sont plus concentrées sur la dimension horizontale et privilégient les valeurs d'amour, d'amitié, le sens de la vie ${ }^{23}$.

Pourtant l'état d'esprit de la personne n'est jamais stable. L'expérience de la vie nous montre qu'indépendamment de tout culte ou croyance la maladie du corps touche aussi l'âme de la personne. En souffrant, la personne se pose beaucoup de questions. Parmi ces questions il y a une qui concerne le sens de la vie, des épreuves et de la mort. Et même si la personne ne se déclare pas religieuse, après un deuil, un accident grave ou une perte d'espoir, elle est en quête de soutien et parfois elle se tourne vers une transcendance, elle l'évoque et la cherche. «L'homme est un animal métaphysique», disait A. Schopenhauer $^{24}$. L'homme «recherche du sacré» ou ce qui est perçu par lui comme

${ }^{21}$ T. CHÂTEL, Prise en compte du spirituel et nouvelles représentations du soin, p. 197.

22 A. COMPTE-SPONVILle, L'esprit de l'athéisme: introduction à la spiritualité sans dieu, Albin Michel 2006, p. 53.

${ }^{23}$ N. SiMARD, Spiritualité et santé, p. 109.

${ }^{24}$ A. SCHOPEnHAUer, Sur le besoin métaphysique de l'humanité, https://www.schopenha uer.fr/fragments/metaphysique-et-religion.html: «Par métaphysique, j'entends tout ce qui a la prétention d'être une connaissance dépassant l'expérience, c'est-à-dire les phénomènes donnés, 
divin et lié à une vérité ou à une réalité ultime ${ }^{25}$. «La quête spirituelle», «la quête du sens», «des solutions face à la souffrance», sont des éléments qui unissent différentes définitions de la spiritualité. À mon avis, face «aux énigmes de l'existence», la science et la raison critique ne peuvent pas toujours suffire à l'homme et ne peuvent pas répondre à toutes ses questions ${ }^{26}$. Et c'est à ce moment que beaucoup de personnes découvrent le monde de la foi et cherche un support à l'expérience religieuse.

L'Association canadienne catholique de la santé (ACCS), définit la spiritualité comme «la lutte que nous menons en vue d'atteindre l'autotranscendance et de rester en rapport avec l'autre» ${ }^{27}$. Je trouve crucial cet aspect interpersonnel dans la dimension spirituelle. Il nous révèle la nature relationnelle de la personne et «fait appel à un sens profond d'être lié à ce qui est» ${ }^{28}$.

La personne par sa nature a besoin de rester en rapport avec l'autre (avec l'autre être humain et avec l'Autre - Dieu). Les relations conjugales, familiales, amicales ainsi que les pratiques religieuses (la méditation, la prière) effectivement aident l'être humain à vivre les épreuves de la vie, passer par la souffrance et même être courageux en face de la mort. Pour plusieurs, le sens et la valeur de la vie prennent racine dans les relations profondes entretenues avec des personnes chères et importantes ${ }^{29}$. Pour que l'intervenant en soins spirituels puisse aider une personne, il faut d'abord établir un contact avec celle-ci. Ce sujet est profondément abordé dans le livre de J.M. Barreau ${ }^{30}$.

En explorant plusieurs livres consacrés aux questions de la spiritualité, nous pouvons facilement constater l'existence de divers types de spiritualités ainsi que diverses expériences spirituelles. Les intervenants en soins spirituels doivent donc respecter ceux qui vivent leur spiritualité liée à la religion, ceux qui vivent la spiritualité sans religion et ceux qui sont en recherche de leurs voies spirituelles. Il faut respecter et essayer de comprendre ce qui est «sacré», c'est-à-dire «le plus important», pour la personne. L'attitude que les intervenants doivent

et qui tend à expliquer par quoi la nature est conditionnée dans un sens ou dans l'autre, ou, pour parler vulgairement, à montrer ce qu'il y a derrière la nature et qui la rend possible».

${ }^{25}$ C. Berghmans, J.-L. Torres, Spiritualité et santé: un pont thérapeutique, p. 45. C.M. PuChalski, Religion, Medicine and Spirituality, p. 45.

${ }^{26}$ C. Berghmans, J.-L. Torres, Spiritualité et santé: un pont thérapeutique, p. 28.

${ }^{27}$ N. SIMARD, Spiritualité et santé, p. 111.

${ }^{28}$ J. PEPIN, Ch. CARA, La réappropriation de la dimension spirituelle en sciences infirmières, p. 34.

${ }^{29}$ P.A. Potter, A.G. Perry, Soins infermières, p. 408.

${ }^{30}$ J.-M. Barreau, Soins Palliatifs. Accompagner pour vivre, Paris, Médiaspaul, p. 282. 
développer par rapport au patient s'exprime dans les mots: être présent, écouter, comprendre, accepter sans jugement et sans objection.

\section{UN LIEN ENTRE LA SPIRITUALITÉ ET LA SANTÉ}

Plusieurs établissent une étroite corrélation entre spiritualité et santé, tandis que d'autres veulent garder ces deux concepts séparés. Mais de plus en plus la spiritualité est reconnue dans le domaine de la santé comme un facteur essentiel dans la prévention et la guérison et surtout dans le cheminement du patient face à la maladie. Selon N. Simard «on accorde un appui croissant à l'idée que les valeurs spirituelles et les buts de la vie apportent une contribution indéniable à la santé physique et psychique ainsi qu'à la satisfaction de vivre» ${ }^{31}$.

Comme j'ai déjà mentionné dans l'introduction, le grand intérêt pour la spiritualité dans le domaine de la santé est lié aux limites de la médecine scientifique qui est capable de soulager des maux physiques, mais souvent elle ne peut pas gérer le problème de la souffrance. Interpellée par les approches thérapeutiques alternatives, la médecine scientifique essaie d'élargir ses notions de santé, de maladie et de guérison et pour cette raison elle s'ouvre aux questions existentielles fondamentales. En voyant la personne dans un sens large et inclusif (corps-âme-esprit) elle perçoit la spiritualité comme une partie intégrante du processus de guérison ${ }^{32}$.

Cette approche n'est pas erronée: les études scientifiques démontrent les effets bénéfiques de la pratique religieuse et de la spiritualité sur la santé. N. Simard dans son article «Spiritualité et santé» estime: «Depuis quelques décennies, les professionnels de la santé, qu'ils soient croyants ou non, ont accordé de plus en plus d'attention au rôle de la spiritualité dans la santé et la guérison. Beaucoup d'études ont démontré l'influence positive de la spiritualité et de la vie spirituelle sur la santé et le bien-être. La majorité des 350 recherches qui, jusqu'à l'an 2000, ont étudié les croyances et la pratique religieuse en lien avec la santé physique ont révélé que les personnes religieuses ou spirituelles sont en meilleure santé physique, qu'elles ont une conduite et un style de vie plus sains et qu'elles font moins appel aux services de santé. Dans le même ordre d'idées, la plupart des 850 études ayant examiné la relation entre la pratique religieuse, la spiritualité et divers aspects de la santé mentale, ont révélé

\footnotetext{
${ }^{31}$ N. SimARD, Spiritualité et santé, p. 114.

${ }^{32}$ N. SimARD, Spiritualité et santé, p. 115.
} 
que les personnes qui ont une forme de pratique religieuse présentent une meilleure santé mentale et font face au stress avec plus de succès» ${ }^{33}$. Même si certains scientifiques ont critiqué ces résultats ${ }^{34}$, je suis persuadée que la spiritualité a un effet positif sur la santé et que le bien-être spirituel peut contribuer à la guérison ou à la quête du sens de la maladie.

Quand quelqu'un est gravement malade il pose souvent la question «pourquoi moi?», «pourquoi maintenant?» Dans sa condition de malade il cherche le sens de sa vie. Souvent, les résultats sont bien surprenants. Par exemple, pour un patient souffrant du cancer, le fait de reconnaître que sa maladie l'a amené à réaliser la valeur et l'importance de relations interpersonnelles serait une façon d'attribuer un sens à sa vie ${ }^{35}$. Il y a beaucoup de témoignages et de preuves que certains patients sont en mesure de comprendre leur maladie en tant que possibilité de croissance, et de voir leur vie et leurs relations d'une nouvelle manière qui leur permet de trouver un sens à leur vie, qui est plus profond et gratifiant qu'avant la maladie ${ }^{36}$. C'est ainsi que la spiritualité peut aider la personne plus que la médecine scientifique. La dernière reconnaît de plus en plus que nos pensées, nos croyances, nos actions, notre façon de voir les choses au quotidien ont une influence capitale sur notre santé.

\section{LE RÔLE PROFESSIONNEL D'UN INTERVENANT, D'UNE INTERVENANTE EN SOINS SPIRITUELS}

Pour bien exercer son rôle professionnel l'intervenant doit être conscient des tâches à accomplir. Dans la description des tâches nous lisons: «l'intervenant exerce des activités de soutien et d'accompagnement à la vie spirituelle et religieuse des patients, à leur famille ainsi qu'à leurs proches. Il planifie, réalise et évalue des activités d'ordre spirituel et religieux. Il effectue des visites d'accueil aux patients et identifie leurs besoins spirituels et religieux. Il répond aux consultations en matière théologique, spirituelle ou éthique, participe aux équipes interdisciplinaires et apporte un support moral aux familles des patients ${ }^{37}$. Je lierais cette description avec ce que j'ai exprimé plus haut: l'attitude à avoir

\footnotetext{
${ }^{33}$ N. SIMARD, Spiritualité et santé, p. 117.

${ }^{34}$ N. SIMARD, Spiritualité et santé, p. 119.

35 P.A. Potter, A.G. Perry, Soins infermières, p. 407.

${ }^{36}$ C.M. PUChALSKI, Religion, Medicine and Spirituality, p. 47.

${ }^{37}$ Intervenant en soins spirituels, http://avenirensante.gouv.qc.ca/carrieres/intervenant-ensoins-spirituels.
} 
par rapport au patient s'exprime dans la présence, l'écoute, la compréhension, et l'acceptation sans jugement et sans objection.

«Exercer des activités et d'accompagnement», à mon avis, c'est être complètement présent avec le patient. La vraie présence s'exprime par des signes d'attention. La personne malade est très sensible. Elle sera capable de distinguer si quelqu'un réalise son rôle professionnel d'intervenant «en mettant tout son cœur» ou «sans cœur». T. Châtel attire l'attention sur cet élément. Il estime que «faire» (i.e. «exercer des activités» selon la description des tâches) est important. Pourtant, comme il souligne, «il ne suffit pas de faire pour prendre soin. L'acte seul, posé de manière mécanique, a parfois conduit à une médecine qu'on a pu qualifier de «déshumanisée», guérisseuse peut-être, mais insuffisamment soignante» ${ }^{38}$. Alors, le rôle professionnel des intervenants devrait s'exercer par la présence qui est une sorte d'offrande de soi. Je suis complètement d'accord avec T. Châtel quand il dit qu'au cœur du soin se tiennent «l'offrande de soi», son temps, sa compassion, son attention, son amour. «Il faut bien comprendre que le principal outil de l'accompagnant, c'est lui-même» ${ }^{39}$.

«Il planifie, réalise et évalue des activités d'ordre spirituel et religieux», selon la description du rôle de l'intervenant en soins spirituels. Mais d'abord on doit connaître la personne, il faut écouter ce qu'elle dit et observer son langage corporel. Parfois la personne exprime plus avec son regard qu'avec ses paroles. Donc, en exerçant son rôle professionnel, l'intervenant doit être attentif. Avant de proposer des activités d'ordre spirituel et religieux, il doit bien comprendre quelle place prend la spiritualité dans la vie d'une personne, quels types d'expériences spirituelles elle a vécues (horizontale, verticale ou les deux), quels évènements ont marqué sa vie, quelles sont ses relations avec ses proches. En écoutant le patient, il peut lui offrir une précieuse possibilité de verbaliser sa perspective, ses inquiétudes et ses besoins.

Avant de répondre à ses besoins en utilisant toutes ses connaissances et ses expériences, un intervenant en soins spirituels doit d'abord les identifier. Cela se fait par la présence attentive et par le dialogue discret. Pourtant, il faut aussi comprendre que certaines situations avec des patients pourront le dé- passer. En ces cas-là, il doit consulter des experts pour trouver la solution adéquate à ces défis. Il est bien de mentionner que même si les intervenants en soins spirituels aient une formation initiale, elle devra être toujours complétée par une formation continue. L'accompagnement de personnes malades ou mourantes exige

38 T. ChâtEL, Prise en compte du spirituel et nouvelles représentations du soin, p. 198.

39 T. CHÂTEL, Prise en compte du spirituel et nouvelles représentations du soin, p. 198. 
beaucoup de travail sur soi-même. La souffrance des autres nous touche et nous convoque au dépassement de nous-mêmes.

C'est pour cette raison que le rôle professionnel des intervenants leur demandera d'explorer leur propre dimension spirituelle. Quand on veut aider les autres au niveau spirituel, quand on veut apporter un support moral aux familles des patients, il faut développer sa propre spiritualité et l'alimenter sans cesse. Selon U. King, «le facteur décisif dans le développement d'une spiritualité personnelle, c'est l'approfondissement de la réflexion et l'honnêteté du regard qu'on jette sur soi-même: l'authenticité qui fait reconnaitre la vérité et accepter l'existence d'une réalité supérieure» ${ }^{40}$.

Pour accompagner les personnes il faut développer et nourrir en soi plusieurs vertus, telles que la patience, la solidarité, l'humilité et l'engagement à la confidentialité. Tous ces mots décrivent bien le quotidien de laccompagnement, mais ils sont d'abord des mots qui caractérisent fondamentalement toutes les voies spirituelles du monde. Il faut donner raison à T. Châtel qu'en ce sens, accompagner est une attitude très exactement spirituelle ${ }^{41}$.

En concluant, nous pouvons évoquer les paroles de Jésus-Christ: «l'homme ne vivra pas de pain seulement...» (Mt. 4,4). En tant qu'unité, corps-esprit-âme, la personne humaine ressent des soifs spirituelles. Le problème du sens en lien avec la finalité de la vie est une question primordiale pour l'homme, même si elle demeure souvent inexprimée. Alors, il est évident que les personnes ont besoin d'être aidées et guidées au niveau spirituel, surtout dans les moments difficiles. C'est la raison pour laquelle je considère que l'existence de la profession des intervenants en soins spirituels au sein de la société laïque est devenue essentielle.

\section{BIBLIOGRAPHIE}

Barreau J.-M., Soins Palliatifs. Accompagner pour vivre, Paris, Médiaspaul 2017.

Berghmans C., Torre J.-L., Spiritualité et santé: un pont thérapeutique, „Intereditions” 2012.

Châtel T., Prise en compte du spirituel et nouvelles représentations du soin, „Médicine palliative", n³, 2007, p. 196-200.

COMPTE-SPONVILLE A., L'esprit de l'athéisme: introduction à la spiritualité sans dieu, Albin Michel 2006.

\footnotetext{
${ }^{40} \mathrm{U}$. KING, La quête spirituelle à l'heure de la mondialisation, p. 25.

${ }^{41}$ T. CHÂtel, Prise en compte du spirituel et nouvelles représentations du soin, p. 199.
} 
Fromaget M., De la distinction âme/esprit, autrement dit du ternaire humain, 2012 https:// www.unidivers.fr/de-la-distinction-ameesprit-autrement-dit-du-ternaire-humain/ (version pdf, p. 1-16).

KING U., La quête spirituelle à l'heure de la mondialisation, Québec 2010.

PEPIN J., CARA Ch., La réappropriation de la dimension spirituelle en sciences infirmières, „Théologiques", vol. 9, n² 2, 2001, p. 33-46.

Potter P.A., Perry A.G., Soins infermières. Fondements généraux, vol. 1, Montréal 2009.

PUCHALSKI C.M., Religion, Medicine and Spirituality: What We Know, What We Don't Know and What We Do, „Asian Pacific Journal of Cancer Prevention”, vol. 11, 2010: MECC Supplement, p.44-50.

SIMARD N., Spiritualité et santé, „Reflets”, vol. 12, n 1, 2006, p. 107-126.

\section{Internet-ressources}

„Corpore et anima unus": la rilevanza etica dell'unità sostanziale dell'uomo all'inizio del terzo millennio, Roma, 24 settembre 2003. http://www.caffarra.it/roma0903.php

CHERBLANC, Jacques et Jobin, GUY, Soutenir le développement du pouvoir d'agir: une composante essentielle des soins spirituels au Québec, „Studies in Religion/Sciences religieuses”, pp. 1-24. Copyright (C) 2020 (Les auteurs). DOI: 10.1177/0008429819893655; https://const ellation.uqac.ca/5462/1/Article\%20Empowerment\%20ISS\%5Bfinal\%5D_sans\%20marque.pdf (23.04.2020).

Définition: psychologie, http://www.psychomedia.qc.ca/lexique/definition/psychologie (22.04. 2020).

Juvénal, Satires, https://sites.google.com/site/texteschoisis/home/juvenal (22.04.2020).

L'histoire en citation, https://www.histoire-en-citations.fr/citations/le-xxie-siecle-sera-spirituel-oune-sera-pas (16.04.2020).

SCHOPENHAUER A., Sur le besoin métaphysique de l'humanité. https://www.schopenhauer.fr/fr agments/ metaphysique-et-religion.html.

Loi sur les services de santé et les services sociaux http://legisquebec.gouv.qc.ca/fr/showdoc $/ \mathrm{cs} / \mathrm{s}-4.2$

\section{DIMENSION SPIRITUELLE EN ÉVIDENCE DANS LE DOMAINE DE LA SANTÉ PAR LA PROFESSION DES INTERVENANTS EN SOINS SPIRITUELS (EXEMPLE DU QUÉBEC)}

\section{RÉSUMÉ}

Beaucoup de sociétés au monde, auparavant profondément chrétiennes, sont devenues laïques dans les dernières décennies. La société québécoise n'est pas une exception, et les raisons pour cela sont diverses. Nous pouvons observer comment les écoles ou les hôpitaux dirigés par les ordres religieux sont devenus la propriété de l'État. L'Église catholique qui auparavant jouait un rôle important dans la société et prenait soin de l'aspect spirituel des personnes, ne joue presque plus ce rôle. Mais cela ne veut pas dire que les besoins spirituels des personnes et leur soif pour la transcendance soient disparus. Les besoins d'un équilibre global de vie se manifestent lors d'une crise causée par la maladie. C'est surtout dans les moments cruciaux, comme la souffrance, la maladie ou la proximité de la mort que les personnes posent beaucoup de questions sur la spiritualité. 
Un aspect positif est que la société laïque du Québec a un respect pour les droits des personnes et de leurs besoins spirituels. Pour répondre à ces besoins, une nouvelle profession a vu le jour et elle prend en compte la dimension spirituelle des personnes. Cette dimension doit être au cœur des pratiques de toutes les personnes engagées à donner un support aux malades. La personne dans son ensemble «corps-âme-esprit» doit être au centre pendant tous les soins médicaux. C'est pour cette raison qu'en premier lieu dans cet article, j'ai parlé de la conception de la personne dans laquelle prévalent l'approche globale et intégrative.

Ensuite, je me suis concentrée sur la notion de la spiritualité. En démontrant la diversité des définitions de la spiritualité, j'ai souligné les éléments communs, tels que la recherche de réponses aux questions : l'identité d'un être humain („qui suis-je?”), le but et le sens de la vie (,pourquoi j'existe?”), le sens de la souffrance et la mort (,pourquoi cela m'arrive?”). Le problème du sens et du but de la vie est une question primordiale pour l'homme, même si elle demeure souvent inexprimée. Beaucoup de gens retrouvent les réponses à ces questions dans la religion, dans leurs rapports avec la transcendance / Être Suprême / Dieu. Certaines personnes, cependant, considèrent la spiritualité dans l'isolement complet de la religion. Ensuite, les principaux éléments de la sphère spirituelle sont souvent les relations avec les proches et les valeurs humaines. Les intervenants en soins spirituels aident les personnes souffrantes à retrouver le sens de la vie, de reconstruire un équilibre de vie global et/ou d'apprendre à «vivre avec» leurs limites. Par leur profession ils offrent l'écoute attentive, la possibilité du dialogue, la solidarité, la compassion et l'amour dont les personnes souffrantes ont tant besoin.

Il est bien de mentionner que les facultés de théologie et les instituts d'études religieuses se sont réorientés pour proposer des programmes académiques afin de former les intervenants en soins spirituels.

Mots clés: vision intégrale de la personne humaine; spiritualité; santé; soutien spirituel; intervenants en soins spirituels; souffrance; maladie; sens de la vie; valeurs humaines.

\section{UWYDATNIENIE WYMIARU DUCHOWEGO W DZIEDZINIE ZDROWIA POPRZEZ ZAWÓD PRACOWNIKÓW OPIEKI DUCHOWEJ (PRZYKŁAD PROWINCJI QUEBEC)}

\section{STRESZCZENIE}

Obserwujemy ogromne zmiany w społeczeństwach, które wcześniej uchodziły za chrześcijańskie. Fala zeświecczenia doprowadziła do tego, że szkoły lub szpitale katolickie, prowadzone kiedyś przez zgromadzenia zakonne, znalazły się w rękach świeckiego państwa z odpowiednio świecką ideologią. Prowincja Quebec nie jest wyjątkiem. Właśnie Kościół Katolicki był tą instytucją, która dbała przede wszystkim o wymiar duchowy człowieka w powierzonych sobie ośrodkach. Opiekę duchową nad pacjentami sprawowali księża, udzielając sakramentów świętych, pocieszając i podtrzymując chorych oraz ich rodziny na duchu. Wskutek zredukowania roli Kościoła poprzez „spokojną rewolucję” i oddzielenie Kościoła od państwa kapelani szpitalni w Quebecu w większości zostali zastąpieni przez pracowników opieki duchowej. Kuriozalne jest zarazem to, że państwo tak laickie jak Kanada w swoich ustawach dotyczących opieki medycznej wymaga poszanowania prawa człowieka i zapewnienia mu opieki duchowej. 
Stąd też funkcjonuje oficjalny zawód opiekuna duchowego, przez co sfera duchowa człowieka zostaje niejako uwydatniona - i to jest rzeczą pozytywną.

W tym artykule zostało podkreślone, że podmiotem opieki duchowej jest zawsze człowiek, postrzegany jako jedność cielesno-psychiczno-duchowa. Sfera duchowa jest trudna do zdefiniowania. Analizując różne definicje duchowości, można wyodrębnić kilka wspólnych elementów, takich jak poszukiwanie odpowiedzi na pytania: o tożsamość człowieka („,kim jestem?”), o cel i sens życia (,po co żyję?”), o sens cierpienia i śmierci (,dlaczego mnie to spotkało?”). Wielu odpowiedzi na te pytania znajduje w religii, a więc w relacji z transcendencją/Siłą Wyższą/Bogiem. Niektórzy jednak rozpatrują duchowość w całkowitym oderwaniu od religii. Wówczas głównymi elementami sfery duchowej często są dla nich relacje z bliskimi oraz wartości ogólnoludzkie. Pracownicy opieki duchowej pomagają człowiekowi cierpiącemu w odnalezieniu sensu życia, odkryciu sensu cierpienia oraz przywróceniu równowagi życiowej poprzez zaakceptowanie ograniczeń i nauczenie się życia z nimi. W tej służbie człowiekowi choremu ważna jest obecność przy nim, aktywne słuchanie, dialog, solidarność, współczucie i miłość. Podkreśla się, że pracownikiem opieki duchowej może być każdy, niezależnie od płci, stanu cywilnego lub religijnej przynależności. Niektóre wydziały teologiczne oraz Instytuty religijne, przeżywające obecnie kryzys z powodu niedoboru studentów na kierunek filozofii czy teologii, po zreorganizowaniu się proponują programy kształcenia dla przyszłych pracowników opieki duchowej.

Słowa kluczowe: integralna wizja osoby ludzkiej; duchowość, zdrowie; wsparcie duchowe; pracownicy opieki duchowej; cierpienie; choroba; sens życia; wartości ludzkie. 